\title{
On Safety Solutions in an Assembly HMI-Cell
}

\section{Rickard Olsen and Kerstin Johansen}

Linköping University

\section{Magnus Engstrom}

Saab AB

CITATION: Olsen, R., Johansen, K., and Engstrom, M., "On Safety Solutions in an Assembly HMI-Cell," SAE Technical Paper 2015-01-2429, 2015, doi:10.4271/2015-01-2429.

\begin{abstract}
The increased diffusion of cooperation between humans and robotics in manufacturing systems is one of the next things to implement within robotics. Since the computer power gets more and more powerful, the possibilities increase to achieve safer working environment, due to that all safety signals demands fast management of data. This could lead to a possibility to work closer and more direct with a robot, using the robot as a third hand. Within an EU FW7 funded project called LOCOMACHs (Low Cost Manufacturing and Assembly of Composite and Hybrid Structures) there are one study focusing on how to support a future higher TRL-leveled HMI cell (Human Machine Interaction) in an assembly task. The main objective in this paper is to present how different external safety systems could support the whole HMI assembly cell to work properly in an industrial context. The scenario for HMI, in this case, is that an operator enters into the robot working area with the aim to perform an assembly task that need two hands. There are different support systems that could be applied to this assembly application, but every one of these needs to coincide with official standards to be applied in an approved industrial HMI assembly cell. The different safety support systems that are used in this research are light barriers, physical barriers and three-position enabler. These safety support systems are tested in a demonstrator assembly cell using a mock-up wing part. The tests with the selected safety support systems in the physical demonstrator will be compared with a concept that uses cameras to achieve the same degree of safety.
\end{abstract}

\section{Introduction}

The diffusion of man and robot is the next big thing in assembly operations and there have been a lot of research in this field the latest years and recently there has released some surveillance equipment for interactions between human and robot [1]. There are different levels of interaction between the human and the machine. Sheridan [2] uses the term Human-Machine Interaction (HMI) to describe all kind of interactions. These could be on such a low level that the human takes all actions and decisions or the highest where the machine is autonomous [2]. In this paper HMI is use to describe when the operator interact with a robot. Here, the Human Robot Collaboration (HRC) is used to show when the operator is using the robot either in a collaborative operation or in a collaborative workspace [ $\underline{3}]$.

Therefore, in the next generation of robots, when implementing Human-Robot collaboration (HRC), the challenges are safety and dependability []].

This paper describes the first physical trials for exploring safety and layout solutions for implementing HRC that have been performed in an EU FW7 project called LOCOMACHs (Low Cost Manufacturing and Assembly of Composite and Hybrid Structures). The results from these trials will be used in order to support the development of a functional HRC-cell for a final demonstrator in the research project. These first trials of robot and manual operation in cooperation in accordance to existing and future valid standards are going to be explored

\section{Theoretical Framework}

There are several ways to define safety, Lowrance [ $\underline{5}]$ define safety, in a valid way, as:

"A thing is safe if its risks are judged to be acceptable"

In a manufacturing working space it is a need to manage safety according to different Standards and regulations, especially when it comes to machines used in the manufacturing process. These standards and regulations aims i.e. to secure the operator from injury. The ISO standard [1] defines a safe state for a machine as:

"Condition of a machine or piece of equipment where it does not present an impendent hazard"

This is important to manage when implementing the next generation of human - machine cooperation solutions. 


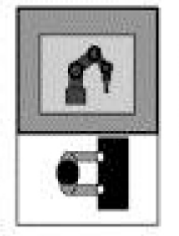

Fence-guarded system

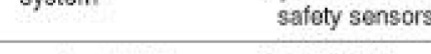

not reachable robot system
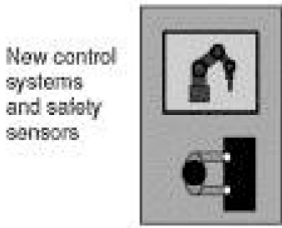

Pre-Colision system: external safoty sensors
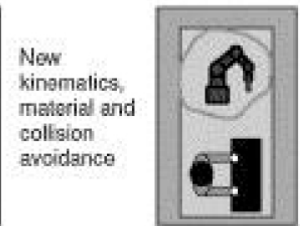

Post-Collision systems: interna safoty control

ach 3 bla by robot system mot sistem

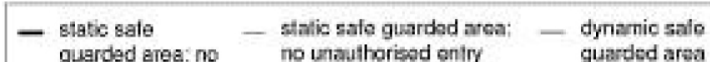
gurdery

Figure 1. Overview of different safety control systems [] $]$

There are different kinds of safety systems, from the classic fenceguarded systems to pre-collision system to post-collision systems, see Figure 1 [6]

The classic fence-guarded systems consist traditionally of a robot with a fence around it. Here, the fences should be designed in order to prevent humans from accessing the robot working area were hazards can occur [ $\underline{3}]$. The fence should prevent any hazards by stopping the robot motion if and when personnel access the safeguarded space [ $\underline{3}]$. Another way to prevent hazard between robot and operator is to utilize a pre-collision system. These systems integrate external support systems with the communication system of a robot to monitoring the workspace [3] . Different kind of sensors might be used for identifying if any personnel are entering the work space, such as vision system or force (weight) sensors on the floor [3]. Finally, the third main type of safety guarding system is the Post-collision systems. Here, integrated sensors, lightweight structures of a robot or software created barriers, are used in order to prevent and/or minimize damage of impact [1].

In ISO there are three major robotic safety standards that apply on these tests, in these three standards they use and cites other standards as well. These other standards are reference document to the three different standards that are identified to be most relevant in this research [3] [7]. One of these standards or the Technical Specification (TS) focusing on Human Robot Collaboration, are under revision and not released yet. So the content in this TS can only be identified and referred to through others research and work in this field of research including interviews with key persons that work in the group that develop the TS. To summaries the process of developing the TS, the robot suppliers are involved in order to get a broad view and anchoring the content in the industry.

To use a robot in collaborative mode a visual indicator is needed and one or more of the following requirements [7]:

- $\quad$ Safety-rated monitored stop

- A category 0 stop, or a decelerated to a category 2 stop but with a safety catch that when it doesn't work it automatically goes into a category 0 stop.

- Hand guiding

- This should be equipped with an emergency stop and an enabling device. And it should operate with a safety-rated monitored speed
- $\quad$ Speed and separation monitoring

- $\quad$ Power and force limiting by inherent design or control

In the robot system, the person and/or company that develop the robotic cell define the different requirements to ensure that the environment in the collaborative workspace is safe. A risk analysis needs to be done, to find all the hazards that could be in this workspace [3] .

\section{Human-Robot Collaboration}

The ISO standards set the safety limits, i.e. speed limits when operator enters the robot working area, for a Human-Robot cooperation setup [1] [ㅁ].

Krüger et al. [1] conclude that man machine cooperation in assembly tasks are needed for more and more flexibility and adaptability, both identified as industry needs. With the help of different support systems, the way of programming can be change in the future; i.e. a simplified way for teaching the robot different paths. The cooperation between man and robot will give new ways on how the industrial design of the robot will be created, such as dual-armed robots or light-weighted robots. Krüger et al. [1] claims that today's focus on accuracy could be changed to safety in the future and support system could help the robot system with both accuracy and safety.

Olsen et al. [ $\underline{8}]$ explained that when the offset printing industry uses small batch sizes it could still use operator centered assembly cells and also use robots, when applying HRC, using the help of support systems. The small batches are solved by the HRC with the help of flexibility [ $\underline{8}]$.

There are several possible set ups for an HRC-cell to be constructed. Three of these are showed below in figure 2 [4].
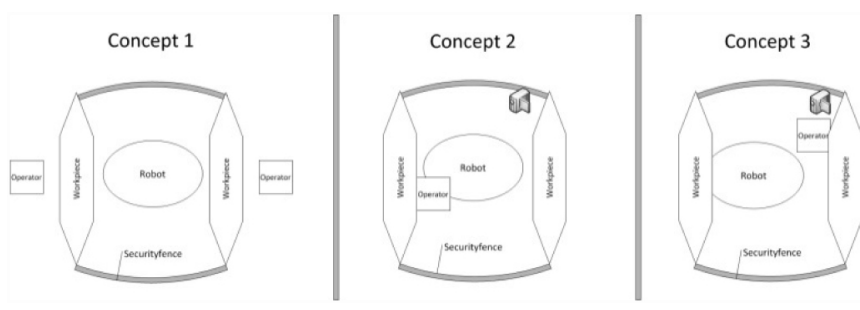

Figure 2. The three Concepts for HRC cells [ $\underline{4}]$

\section{Support Systems}

A three-dimensional vision sensor and a force sensor are two different kinds of support systems [9]. With the three-dimensional vision sensor search for positional errors could be done, it is sensitive for motion and/or parts that is observed in a $3 \mathrm{D}$ volume and which posture of the part. The computer that manages this receives images from the vision system and then with help of algorithms creates a 3D environment of the vision systems subjected area [9] [10]. When an object enters the subjected 3D environment the vision system should react, dependent on how close the new object is to the robot and what kind of speed vector it has, in different ways. On the signal from the vision system the reaction that the robot will do could be either change path, make a category 0 stop or lower its speed, depending on the situation [10]. Force sensors are used either as an external or internal sensor at the robot that controls that it does not crash onto an object i.e. a product, operator or other new objects that has entered the robots projected movement [9]. Impact test of different kind, such 
as the Head Injury Criterion (HIC) test, have evaluated robot systems limitations and potential in relation to injury and velocity [] [ㅇ]. This gives indications that velocity up to $2 \mathrm{~m} / \mathrm{s}$ on the robot is below today's limit according to robot impact test compared with impact test in the automotive industry []. This exemplifies Jonsson et. al. [11] shown in an assembly application that uses an external force sensor. Here, they used a force sensor controlled with Matlab Stateflow; to create a production cell that is more flexible and cheaper than a production cell that uses dedicated equipment and jigs, see Figure 3.

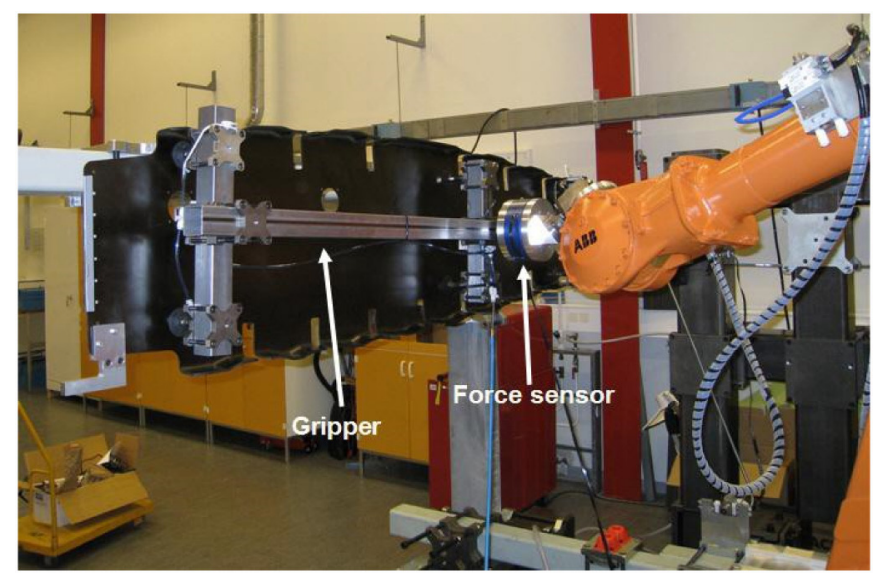

Figure 3. A force sensor controlled robot used in a conceptual flexible assembly cell for rib assembly [11]

\section{TRL - Technology Readiness Level}

Technology Readiness Level (TRL) is according to Mankins [12] a measurement that assesses the maturity and helps to do comparison between technologies. The levels grades from 1 to 9 , with 9 as the most mature technology level [12]. Roughly the levels can be described as follows: the lower levels, 1-3, are identified as covering different basic research issues, the level 4-6 are pre-industry implementation levels, and the levels 7-9 are different levels for a technology implemented in industry.

\section{The Test Setup}

The preparation of the test setup for the demonstrator of the HMI assembly cell included some interviews and benchmarking regarding safety and regulations. Based on an interview with one key person with deep knowledge about the work with the future Technical Specification (TS) it was clearly stated that the TS will contain a more detailed description about collaborative robot systems including the workplaces. Therefore, the demonstrator cell will test two different safety set ups.

In the first setup the robot performs the movement with the component to be assembled (here: the rib) and then the robot stops, thereafter the operator enters the working space and perform the assembly task, which means that the safety solution is less complex since the robot is stopped and in a controlled position. In the second set up the robot and the operator share the same working area at the same time, i.e. the robot can move while the operator is close to it. This is however more demanding from a safety point of view and require more complex support systems to achieve a safe environment. The test cell designed in this paper is arranged according to Concept 2 in figure 1 [4]. This layout can be used for testing both set-ups described:

1. Robot positioned the rib and stop - the operator enters the cell and performs assembly tasks, while the robot holds the rib in position.

2. The robot and operator share the same working area while the robot is able to move at the same time as the operator perform assembly tasks.

\section{Cell Setup}

In this research a physical demonstrator cell was developed for evaluation of different safety solutions. As shown in figure 4, the robot used is a Yaskawa SDA10, but in the demonstrator only one arm was used. A custom made vacuum gripper was available, developed during another research project, for gripping the rib. The rib and the wing part were in this case a mock-up manufactured in a wooden material in order to achieve a lifelike experience of the size and reachability when the robot and an operator should share the same area. The wing part of the mock-up was placed in a fixture made of the reconfigurable Box-Joint, shown in figure 8. Future improvements of the demonstrator setup are better fixtures, the strong back and the connections between the first safety system and the robot, inter alia.

The demonstrator cell is evaluating different safety solutions both physically and virtually:

1. Camera safety solution (physically)

2. Reachability between operator and robot (physically)

3. Light curtain safety (virtually and physically)

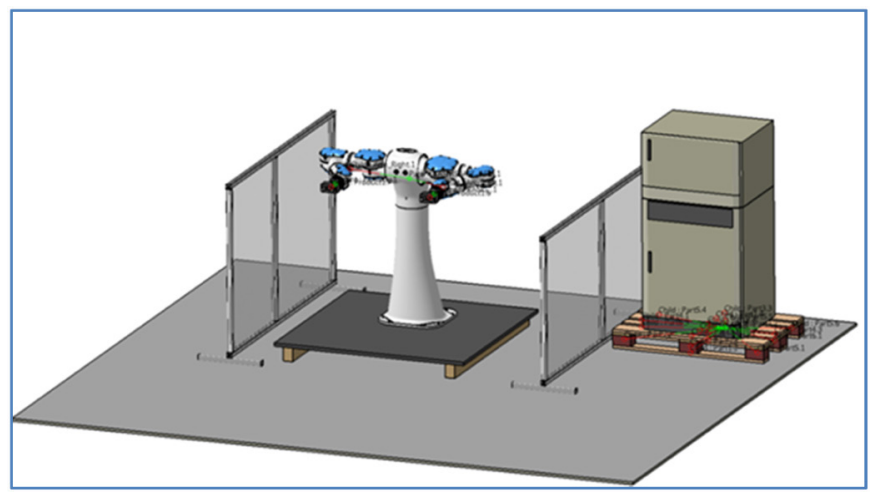

Figure 4. CAD-drawing over part of the demonstrator cell

\section{Mock Up}

A mock up is built in wooden material to represent a part of an aeroplane wing that is used in this research project. However, this mock up is not hundred per cent accurate but it support the demonstrator needs to evaluate safety in a HMI perspective. The rib posts have the correct contour according to the final demonstrator in the overall research project, figure 5. Skins and spars are built to fit the ribs and do not have the right measurements, here this is not needed since the aim of the demonstrator is related to evaluating issues related to HMI. 


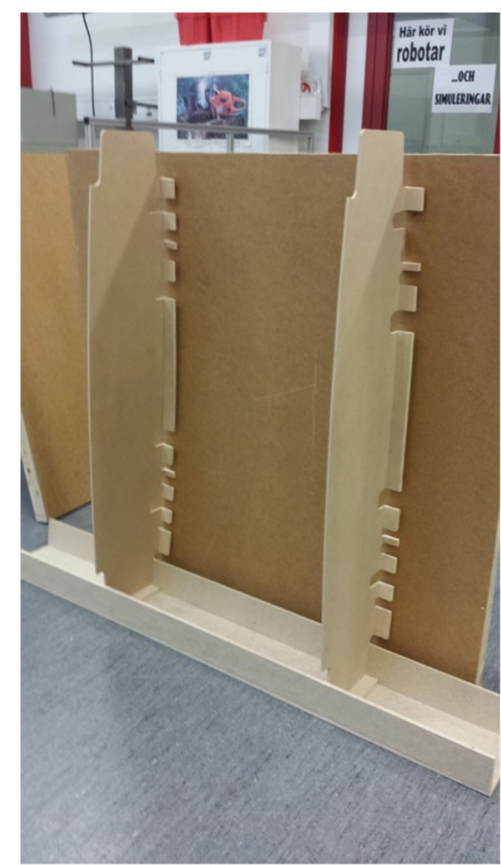

Figure 5. Some of the parts of the wooden mock up for the wing part

\section{Kinect Solution}

As a sub-project in this demonstrator development, a study on safety solution using Kinect as a safety monitoring equipment has been performed. This equipment are monitoring the area around the robot, locating moving objects and calculates the closest point of the moving object and displaying it at an display, figure 6. It uses different colour codes on a flat screen to visual if the object is to close to the robot or not, red for to close, yellow for collaborative mode and green for normal mode. The system is controlled by Industrial ROS, which is an open source program for robot programming.

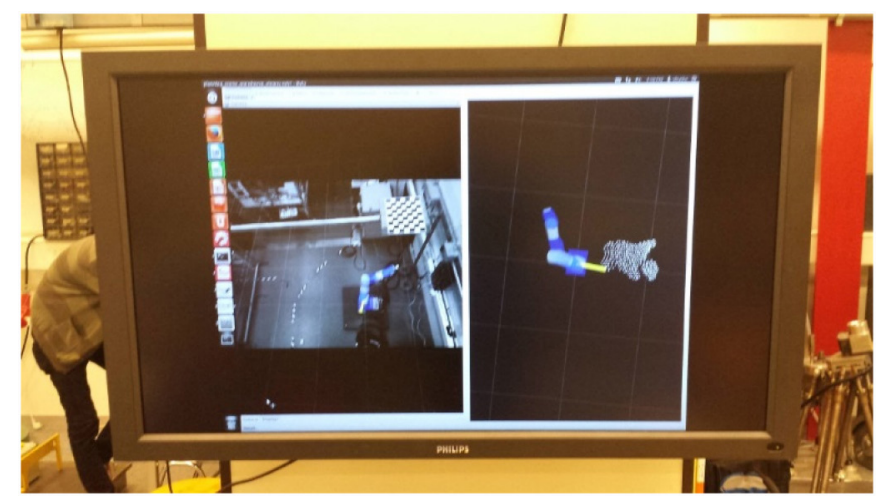

Figure 6. The flat screen visualization of the Kinect safety monitoring

\section{Virtual Cell}

In the virtual cell, the use of light curtains is demonstrated. These light curtains are placed horizontal approximately $300 \mathrm{~mm}$ over the floor and in the position that it covers the maximum space that is needed for a safe environment, to avoid harmful collisions between the operator and the robot with its work piece. The choice to use 300 $\mathrm{mm}$ over the floor is in considerations of a jumping human and that it is at the same time nearly impossible for an adult to crawl under the light curtain. Compare to classic fence-guard system this gives a more transparent overview of the work area. A small fixture holds the light curtains in the right position. A controller is placed so the operator needs to leave the working area and push a button as an extra safety feature. This controller gives the robot and safety system a signal when the operator is finished with the operation and an emergency button is also placed on it, figure 7 .

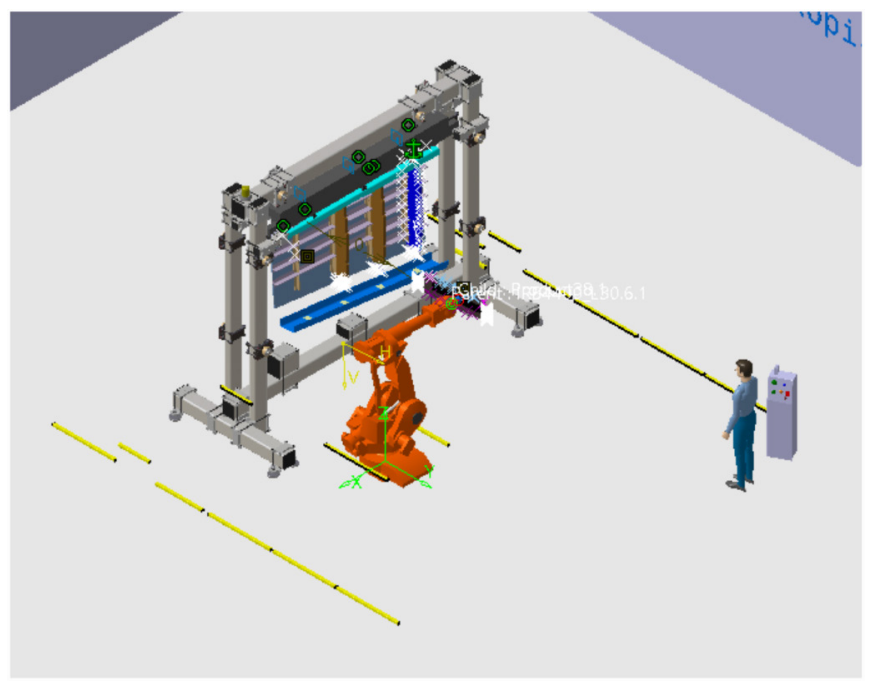

Figure 7. The virtual cell developed for the demonstrator cell of HMI

\section{Discussion}

The demonstrator parts are based on trials testing the different components one by one in order to evaluate their performance to match the requirements for completing a total test of all technologies together. Some of the components has been tried together; the mock up and the robot cell has been evaluated together in order to check the reachability of all points on the rib. It was also tried to see if the operator could reach different things, such as positions for assembly, in the cell when the robot are placed in different positions. It was obvious that the reachability in combination with safety solutions that maximize the productivity is a vital variable when implementing HMI. Figure 8 and 9 below present how the robot positioning the rib in relation to the free space there is for an operator to use. In the used demonstrator assembly cell the mock-up is based on an early design in the overall research project. Therefore, our evaluations are based on experiences where the robot grips the rib from "the wrong side" compare to the final solution in the overall project. However, this does not affect the results about safety in the HMI solution The first test was positive; the reachability and flexibility of the robot was adequate for the task.

The Kinect solution showed that with a cheap and open source programming solution (ROS) it is possible to achieve a safety monitoring system. However, it is important to secure that the ROS is comparative with the selected robot and safety equipment. The developed tracking algorithm observe and visualize properly when one object entering the Kinects collaboration area, but when two or more objects enter the collaborative area the computer have problem to process all data in real time. Hence, the need for proper computer capacity is identified as important when implementing a camera safety solution. The different color coding in the visualization of the Kinect data signals to the operator the actual robotic system placement. If connected to the robot system as an active safety system, the red symbolize a safety-rated stop, the yellow is used to indicate that the speed of the robot should be set into collaborative speed and finally, green is a signal to the system to use full speed. 


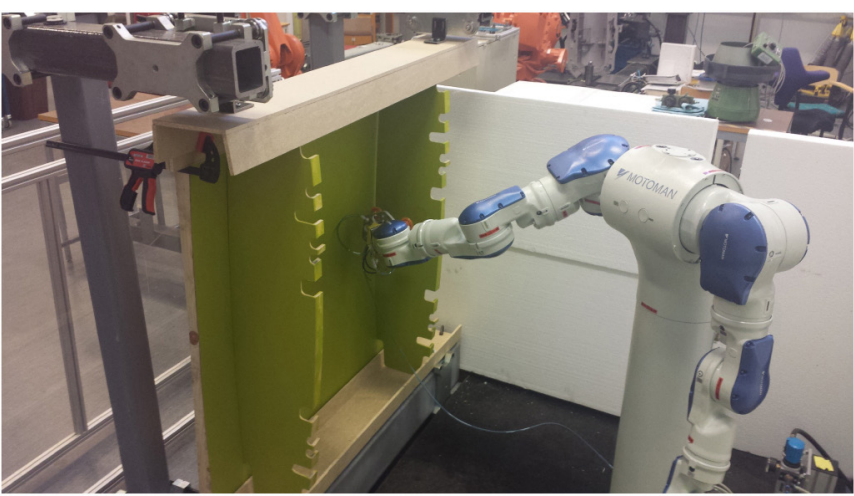

Figure 8. Positioning of the rib by the robot in the physical demonstrator of the test cell

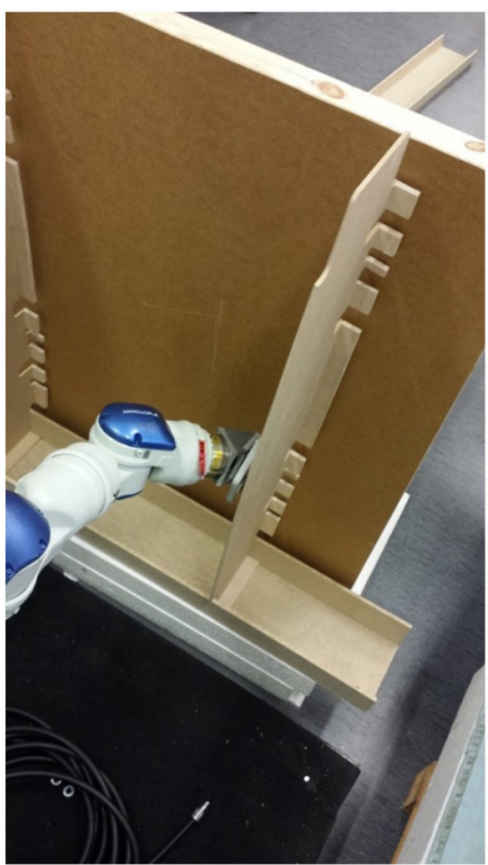

Figure 9. Testing position of sharing work space within the robot cell

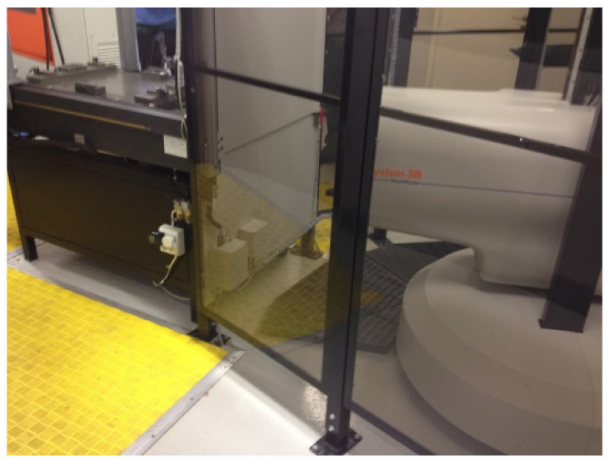

Figure 10. Safety mats with a fenced machine in an industrial process

The virtual cell uses light curtains as a safety solution, figure 7. This was chosen because the alternative, safety mats, was more expensive and does not have as high safety classification as light curtains; to be used in an industrial environment. Furthermore, safety mats needs to cover a huge area of the floor if it should work without physical fences; figure 10 illustrates the need of mats with physical fences. Therefore, light curtains are, as of today, considered to be more cost effective.
The different evaluation trials about safety and layout solutions for a future HRC assembly cell needs to be related to the aimed TRLlevels in the overall project. Since the Technical Specification (TS) not yet are released and the TRL level that is aimed for in the LOCOMACHs project is TRL 5 or 6 , our results must be related to a flexible implementation of HRC. The TRL-levels in the overall project are defined in figure 11 as:

- TRL 5 - small-scale partial integration of technology (with partially representatives interfaces)

- $\quad$ TRL 6 - large-scale major integration, highly representative implementation of technology in target elements

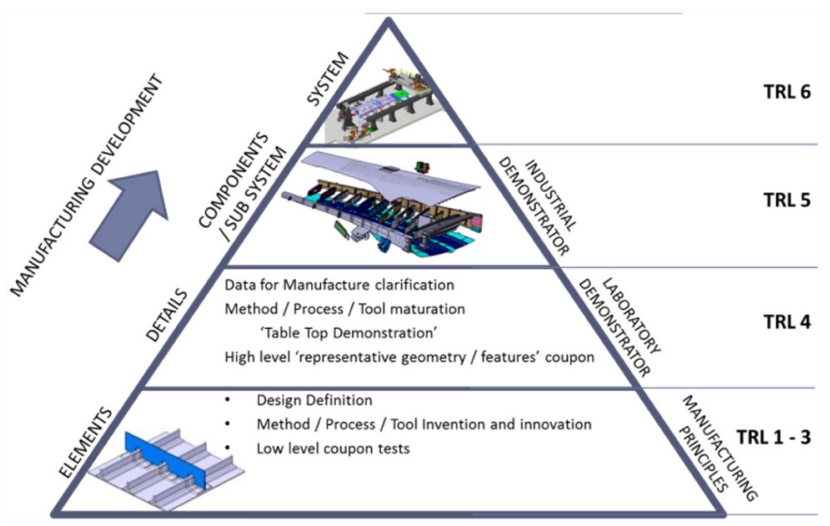

Figure 11. The LOCOMACHs projects defined TRL-levels

The different trials in the demonstrator cells in this paper have different TRL-level and different possibility to reach higher TRLlevels. The physical cell setup in the lab should have reached TRL 5 if it was not that the TS are not released yet, so it could only reach TRL 4. Here, the safety solution is TRL 4. The virtual cell has reached TRL 3 when it comes to HRC. However, to convert the virtual cell that visualizes a HMI-cell now, into an HRC-cell could be done. The transition of the virtual HMI-cell into a HRC-cell includes adding an enabling switch, a 3-stage safety button, and a camera safety solution, i.e. the Kinect solution. The Kinect solution that has been developed and evaluated here has, according to our overall projects TRL definitions, reached a TRL 4.

\section{Summary/Conclusions}

Based on the performed trials in this demonstrator cell equipment and technology on the market in combination with some custom made components can be combined into a HRC-cell. This has been evaluated in different steps combining virtual and physical evaluations. It is solvable to secure safety for the operator when the robot move, however, it is crucial to secure safety when the operator and the robot share the same working area. The solution must combine several different support systems and this will demand computer capacity and redundant system solutions. Furthermore, an enabling device must be used according to the old robot safety standards and preliminary information about the content of the TS and the vision solution is there to slow down or stop the robot if the operator gets to close. There can also be a support to use force sensors to avoid harm if the robot should collide with the operator, but this is suggested to be part of the redundant solution in the first 
implementation phases of HRC in assembly. Future research is to build and test a physical HRC cell for a rib assembly in the aerospace industry in a complete demonstrator combining these results.

The risk for implementing HRC in near future is related to the release of the new TS. This research is therefore focusing on several different supporting systems, in order to have knowledge about the needs and possibilities to implement HRC stepwise. One solution is based on the existing standards, the physical cell setup, and one that is relevant for the future, the virtual cell. This stepwise implementation could gain the technology implementation in the future, since the operators are learning step by step how to collaborate with a robot in a safe way.

The main conclusion is that safety solutions, which are reliable and not reducing productivity, will be the key for implementing HRC in industrial assembly cells.

\section{References}

1. Krüger, J., Lien, T. K. and Verl A., "Cooperation of human and machines in assembly lines," CIRP Annals - Manufacturing Technology, 58, 628-646, 2009.

2. Sheridan, T.B., Humans and Automation - System Design and Research Issues (New York: Wiley, 2002).

3. SIS., Robots and robotic devices - safety requirements for industrial robots - part 2: Robot systems and integration (EN ISO 10218-2:2011), European committee for standardization, 2011.

4. Olsen, R., and Johansen, K., "Assembly cell concept for human and robot in cooperation," The 22th International Conference on Production Research., Iguassy Falls (BRA), 2013.

5. Lowrance, W. W., "Of acceptable risk: science and the determination of safety," Calif William Kaufmann Inc., 1976.
6. Oberer, S. and Schraft, R-D., "Robot-dummy crash tests for robot safety assessment,” IEEE I.C.R.A Conf., Roma (ITA), 2934-2939, 2007.

7. SIS., Robots and robotic devices - safety requirements for industrial robots - part 1: Robots (EN ISO 10218-1:2011), European committee for standardization, 2011.

8. Olsen, R., Johansen K. and Jonsson M., "Flexible automation in the printing industry," The 5th International Swedish Production Symposium., Linköping (SWE), 45-51, 2012.

9. Sakakibara, S., "The latest robot systems which reinforce manufacturing sector," IEEE International Conference on Robotics and Automation, Taipei, 2878-2883. 2003.

10. Sahr, B., Buttrick, J., Munk, C. and Bossi R., "Aircraft manufacturing and assembly," Springer Handbook of Automation (Nof S.Y. (Ed)), Springer, Berlin, 893-910, 2009.

11. Jonsson, M., Stolt, A., Robertsson, A., Murray, T. et al., "Force Controlled Assembly of a Compliant Rib," SAE Technical Paper 2011-01-2734, 2011, doi:10.4271/2011-01-2734.

12. Mankins, J.C., "Technology Readiness Levels - A White Paper," NASA, 1995

\section{Acknowledgments}

The research leading to these results has received funding from the European Union's Seventh Framework Programme (FP7/2007-2013) under grant agreement $\mathrm{n}^{\circ} 314003$.

The sub-project members in the Kinect work, Olle Fridolfsson, Niklas Hansson, Patrik Hillgren, Benjamin Ingberg, Pär Lundgren and Mattias Nilsson, are acknowledged.

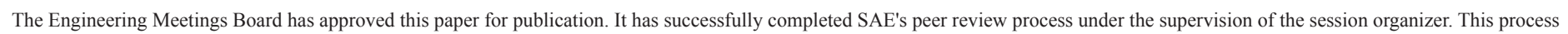
requires a minimum of three (3) reviews by industry experts.

This is a work of a Government and is not subject to copyright protection. Foreign copyrights may apply. The Government under which this paper was written assumes no liability or

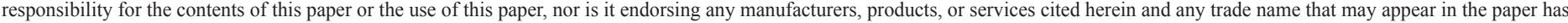
been included only because it is essential to the contents of the paper.

Positions and opinions advanced in this paper are those of the author(s) and not necessarily those of SAE International. The author is solely responsible for the content of the paper.

ISSN 0148-7191

http://papers.sae.org/2015-01-2429 